\title{
Shear Stress for Homann and Convergent Flows Arising in the Boundary Layer Theory with Odd Decimal Numbers of Tangential Velocity
}

\author{
Mamun Miah", Abul Kalam Azad, Masidur Rahman \\ Department of Applied Mathematics, University of Rajshahi, Rajshahi, Bangladesh
}

Email address:

mamun0954@gmail.com (M. Miah), azad267@gmail.com (A. K. Azad), masidur09@gmail.com (M. Rahman)

To cite this article:

Mamun Miah, Abul Kalam Azad, Masidur Rahman. Shear Stress for Homann and Convergent Flows Arising in the Boundary Layer Theory with Odd Decimal Numbers of Tangential Velocity. Applied and Computational Mathematics. Vol. 5, No. 1, 2016, pp. 23-29.

doi: 10.11648/j.acm.20160501.14

\begin{abstract}
In this paper, we discussed the effect of shear stress for Homann and Convergent flows arising in the boundary layer theory with odd decimal numbers of tangential velocity. By this study we have to discuss positive solution, Homann flow, convergent flow, shear stress, tangential velocity etc. From beginning to end of the study, we have compared of stresses of different fluid flows arising in the boundary layer theory. The resulting figure is compared with the previous figure which was obtained by many authors.
\end{abstract}

Keywords: Shear Stress, Homann Flow, Convergent Flow, Boundary Layer Flow

\section{Introduction}

Any real fluids moving along solid boundary will sustain a shear stress on that boundary. The no-slip condition dictates that the speed of the fluid at the boundary is zero, but at some height from the boundary the flow speed must equal that of the fluid. The region between these two points is aptly named the boundary layer. For all Newtonian fluids in laminar flow the shear stress is proportional to the strain rate in the fluid where the viscosity is the constant of proportionality. However, for non-Newtonian fluids, this is no longer the case as for these fluids the viscosity is not constant. The shear stress is imparted onto the boundary as a result of this loss of velocity. The existence of positive solution for the singular second-order nonlinear boundary value problem arising in the boundary layer theory for the strong suction is studied. Some of researchers as Schmidt [1], Soewono et al [2], Vajravelu et al [3], Schlichting $\mathrm{H}$ [5] etc are discussed the boundary layer theory. Singular nonlinear differential equations arising in the Homann flow are discussed by Shin [1997]. Molla and Banu [7] studied Some singular nonlinear BVPS arising in the boundary layer flow. Molla and Banu [9] shown that existence and uniqueness of positive solution of a singular nonlinear BVP. For a flat plate a singular non-linear BVP arising in the boundary layer flow studied by Molla[10]. Also a singular non-linear boundary value problem arising in a convergent channel was done by Molla [12]. The method of finding positive solution arising in the boundary layer theory by several authors; see Molla M. R. [8], Molla M. R. and M. Begum [13], M. R. Molla et al [11]. The effects of the positive solution arising in the boundary layer theory are discussed for the case of even decimal number of tangential velocity in the paper of Molla [15]. We have solved this problem by using the constructive method such as the method of upper and lower solutions with odd decimal number of tangential velocity to establish the existence of positive solution of (16). We have also tried to compare the result with the result of Molla [15] for even decimal number of tangential velocity. In this paper we have also tried to compare the shear stress of the strong suction with the shear stress of the Homann and Convergent flow. Obtained result which is almost same as obtained result by Molla [15].

\section{Formulation of the Problem}

The differential equation

$$
\begin{aligned}
& \mathrm{u} \frac{\partial u}{\partial x}+\mathrm{v} \frac{\partial u}{\partial y}=\mathrm{U} \frac{d U}{d x}+v \frac{\partial^{2} u}{\partial y^{2}} \\
& \frac{\partial u}{\partial x}+\frac{\partial v}{\partial y}=0
\end{aligned}
$$

With boundary conditions 


$$
\begin{gathered}
y=0: u=v=0 \\
y \rightarrow \infty: u=U
\end{gathered}
$$

is known as the boundary-layer equations for plane steady incompressible flow.

The continuity equation can be integrated by introducing the stream function $\Psi(\mathrm{x}, \mathrm{y})$ with

$$
u=\frac{\partial \Psi}{\partial y} v=-\frac{\partial \Psi}{\partial x}
$$

The equation (1) becomes,

$$
\frac{\partial \Psi}{\partial y} \frac{\partial^{2} \Psi}{\partial x \partial y}-\frac{\partial \Psi}{\partial x} \frac{\partial^{2} \Psi}{\partial y^{2}}=\mathrm{U} \frac{d U}{d x}+v \frac{\partial^{3} \Psi}{\partial y^{3}}
$$

With the boundary conditions

$$
\begin{gathered}
\frac{\partial \Psi}{\partial y}=\frac{\partial \Psi}{\partial x}=0 \text { for } \mathrm{y}=0 \\
\frac{\partial \Psi}{\partial y}=\mathrm{U} \text { for } \mathrm{y} \rightarrow \propto
\end{gathered}
$$

We now carry out a coordinate transformation from the variable $\mathrm{x}, \mathrm{y}$ to the new dimensionless variables

$$
\begin{gathered}
\xi=\frac{x}{l}, \eta=\frac{y}{l} \cdot \sqrt{\operatorname{Re}} / \delta(\xi) \\
\mathrm{f}(\xi, \eta)=\Psi(\xi, \eta) \sqrt{\operatorname{Re}} /\left(l U_{N}(\xi) \delta(\xi)\right)
\end{gathered}
$$

Also we have $\operatorname{Re}=V l / v$ where $\mathrm{Re} \rightarrow \operatorname{Reynolds}$ numder, $V \rightarrow$ Reference velocity, $1 \rightarrow$ Reference length, $(\xi) \rightarrow$ boundary layer.

From (3) we have

$$
\begin{aligned}
& \Psi(\xi, \eta)=l U_{N}(\xi) \delta(\xi) \mathrm{f}(\xi, \eta) / \sqrt{R e} \\
& \left.\therefore U_{N} f^{\prime}\left\{1 / 1 .\left(U_{N}^{\prime} f^{\prime}+U_{N} \frac{\partial f^{\prime}}{\partial x}\right)-U_{N} f^{\prime \prime} \mathrm{y} \sqrt{\operatorname{Re}} \delta^{\prime} /\left(l^{2} \delta^{2}\right)\right)\right\}-\left[1 / \sqrt{\operatorname{Re}}\left\{U_{N} \delta \frac{\partial f}{\partial \xi}+f \frac{\partial}{\partial \xi}\left(U_{N} \delta\right)\right\}-U_{N} y \delta^{\prime} f^{\prime} /(l \delta)\right] \frac{\sqrt{\operatorname{Re}} U_{N}}{l \delta} f^{\prime \prime}= \\
& \frac{U}{l} \frac{d U}{d \xi}+v \operatorname{Re} U_{N} /\left(l^{2} \delta^{2}\right) f^{\prime \prime \prime} \\
& \Rightarrow U_{N} U_{N}{ }^{\prime} f^{\prime 2} / l+U_{N}{ }^{2} f^{\prime} \frac{\partial f^{\prime}}{\partial x} / 1-U_{N}{ }^{2} \mathrm{y} \frac{\sqrt{\operatorname{Re}} \delta^{\prime} f^{\prime} f^{\prime \prime}}{l^{2} \delta^{2}}-\frac{U_{N}{ }^{2} f^{\prime \prime} \frac{\partial f}{\partial \xi}}{l}-\frac{U_{N}}{l \delta} f f^{\prime \prime} \frac{\partial}{\partial \xi}\left(\delta U_{N}\right)+U_{N}{ }^{2} \mathrm{y} \frac{\sqrt{R e} \delta^{\prime} f^{\prime} f^{\prime \prime}}{l^{2} \delta^{2}}=\frac{U}{l} \frac{d U}{d \xi}+v R e U_{N} /\left(l^{2} \delta^{2}\right) f^{\prime \prime \prime} \\
& \Rightarrow v \frac{R e U_{N}}{l^{2} \delta^{2}} f^{\prime \prime \prime}+\frac{U_{N}}{l \delta} f f^{\prime \prime} \frac{\partial}{\partial \xi}\left(\delta U_{N}\right)+\frac{U}{l} \frac{d U}{d \xi}-\frac{U_{N} f^{\prime 2}}{l} \frac{d U_{N}}{d \xi}=\frac{U_{N}^{2}}{l} f^{\prime} \frac{\partial f^{\prime}}{\partial x}-\frac{U_{N}{ }^{2} f^{\prime \prime} \frac{\partial f}{\partial \xi}}{l} \\
& \Rightarrow \mathrm{V}_{U_{N}} /\left(l \delta^{2}\right) f^{\prime \prime \prime}+\frac{U_{N}}{l \delta} f f^{\prime \prime} \frac{\partial}{\partial \xi}\left(\delta U_{N}\right)+\frac{U}{l} \frac{d U}{d \xi}-\frac{U_{N} f^{\prime 2}}{l} \frac{d U_{N}}{d \xi}=\frac{U_{N}^{2}}{l} f^{\prime} \frac{\partial f^{\prime}}{\partial x}-\frac{U_{N}^{2} f^{\prime \prime} \frac{\partial f}{\partial \xi}}{l} \\
& \Rightarrow f^{\prime \prime \prime}+l \delta^{2} /\left(\mathrm{V} U_{N}\right) \frac{U_{N}}{l \delta} f f^{\prime \prime} \frac{\partial}{\partial \xi}\left(\delta U_{N}\right)+l \delta^{2} /\left(\mathrm{V} U_{N}\right) \frac{U}{l} \frac{d U}{d \xi} l \delta^{2} /\left(\mathrm{V} U_{N}\right) \frac{U_{N} f^{\prime 2}}{l} \frac{d U_{N}}{d \xi}=l \delta^{2} /\left(\mathrm{V} U_{N}\right) \frac{U_{N}{ }^{2}}{l}\left(f^{\prime} \frac{\partial f^{\prime}}{\partial x}-f^{\prime \prime} \frac{\partial f}{\partial \xi}\right) \\
& \Rightarrow f^{\prime \prime \prime}+\frac{\delta}{V} \frac{\partial}{\partial \xi}\left(\delta U_{N}\right) f f^{\prime \prime}+\frac{U}{U_{N}} \frac{\delta^{2}}{\mathrm{~V}} \frac{d U}{d \xi}-\frac{\delta^{2}}{\mathrm{~V}} \frac{d U_{N}}{d \xi} f^{\prime 2}=\frac{\delta^{2} U_{N}}{V}\left(f^{\prime} \frac{\partial f^{\prime}}{\partial x}-f^{\prime \prime} \frac{\partial f}{\partial \xi}\right) \\
& \Rightarrow f^{\prime \prime \prime}+\alpha_{1} f f^{\prime \prime}+\alpha_{2}-\alpha_{3} f^{\prime 2}=\frac{\delta^{2} U_{N}}{V}\left(f^{\prime} \frac{\partial f^{\prime}}{\partial x}-f^{\prime \prime} \frac{\partial f}{\partial \xi}\right)
\end{aligned}
$$

Where, $\alpha_{1}=\frac{\delta}{V} \frac{\partial}{\partial \xi}\left(\delta U_{N}\right), \alpha_{2}=\frac{U}{U_{N}} \frac{\delta^{2}}{\mathrm{~V}} \frac{d U}{d \xi}, \alpha_{3}=\frac{\delta^{2}}{\mathrm{~V}} \frac{d U_{N}}{d \xi}$

The similar solution exists only when $f$ and $f^{\prime}$ does not depends on $\xi$. $\therefore$ The equation (6) becomes 


$$
f^{\prime \prime \prime}+\alpha_{1} f f^{\prime \prime}+\alpha_{2}-\alpha_{3} f^{\prime 2}=0
$$

With boundary conditions

$$
f(\eta)=f^{\prime}(\eta)=0 \text { at } \eta=0
$$

and

$$
f^{\prime} \rightarrow 1 \text { as } \eta \rightarrow \infty
$$

When $\alpha_{1}=\alpha$ and $\alpha_{2}=\alpha_{3}=\beta$ i. e. $U=U_{N}$ Than equation (7) becomes

$$
f^{\prime \prime \prime}+\alpha f f^{\prime \prime}+\beta\left(1-f^{\prime 2}\right)=0
$$

With boundary conditions

$$
f(\eta)=f^{\prime}(\eta)=0 \text { at } \eta=0
$$

and

$$
f^{\prime} \rightarrow 1 \text { as } \eta \rightarrow \infty
$$

This equation (8) is known as Falkner-Skan boundary layer equation.

When $\alpha=1$ and $\beta=0.5$, the equation ( 8 ) becomes

$$
f^{\prime \prime \prime}+f f^{\prime \prime}+0.5\left(1-f^{\prime 2}\right)=0
$$

With boundary conditions (9). This equation represents Homman flow.

For $\alpha=0$ and $\beta=1$, the equation (8) takes the following form

$$
f^{\prime \prime \prime}+1-f^{\prime 2}=0
$$

With boundary conditions (9). This equation represents flow in a convergent channel.

Shin [4] discussed the differential equation (10) with boundary conditions (9) by using the method of upper and lower solutions. Later Molla [15] used this method for the differential equation

$$
\varphi^{\prime \prime \prime}+\varphi^{\prime \prime}=0
$$

With boundary conditions

$$
\begin{array}{r}
\varphi(\eta)=\varphi^{\prime}(\eta)=0 \text { at } \eta=0 \\
\varphi^{\prime} \rightarrow \infty \text { as } \eta \rightarrow \infty
\end{array}
$$

which arises in the boundary layer theory for strong suction.

Now we want to discuss the constructive method such as, the method of upper and lower solutions with graphically by the help of Molla [15].

Let us take the shear stress $\mathrm{h}(\mathrm{x})=\varphi^{\prime \prime}(\eta)$ and the tangential velocity $\mathrm{x}=\varphi^{\prime}(\eta)$ as dependent and independent variable respectively. The quantity $\mathrm{x}$ and $\mathrm{h}$ are called Crocco variable.

Now

$$
\begin{gathered}
h^{\prime}=\frac{d h}{d x}=\frac{\frac{d h}{d \eta}}{\frac{d x}{d \eta}}=\frac{\frac{d\left(\varphi^{\prime \prime}\right)}{d \eta}}{\frac{d\left(\varphi^{\prime}\right)}{d \eta}}=\frac{\varphi^{\prime \prime \prime}}{\varphi^{\prime \prime}}, \\
\text { e. } h^{\prime} \varphi^{\prime \prime}=\varphi^{\prime \prime \prime}
\end{gathered}
$$

Differentiating (14) with respect to $\eta$ we get

$$
\begin{gathered}
\varphi^{\prime \vee}=\frac{d}{d \eta}\left(h^{\prime} \varphi^{\prime \prime}\right) \\
=\frac{d x}{d \eta} \frac{d}{d x}\left(h h^{\prime}\right) \\
=\varphi^{\prime \prime}\left\{\mathrm{h} h^{\prime \prime}+\left(h^{\prime}\right)^{2}\right\} \\
=\mathrm{h}^{2} h^{\prime \prime}+\mathrm{h}\left(h^{\prime}\right)^{2}
\end{gathered}
$$

Again differentiating (12) we get

$$
\begin{gathered}
\varphi^{\prime v}+\varphi^{\prime \prime \prime}=0 \\
\Rightarrow \mathrm{h}^{2} h^{\prime \prime}+\mathrm{h}\left(h^{\prime}\right)^{2}+\mathrm{h} h^{\prime}=0 \text { [using equation (14)] } \\
\Rightarrow \mathrm{h} h^{\prime \prime}+\left(h^{\prime}\right)^{2}+h^{\prime}=0
\end{gathered}
$$

Now

$$
\mathrm{h}(\mathrm{x})=\varphi^{\prime \prime}=\frac{d}{d \eta}\left[\varphi^{\prime}(\eta)\right]
$$

Therefore,

$$
\mathrm{h}(1)=\left[\frac{d}{d \eta}\left(\varphi^{\prime}(\eta)\right)\right]_{\mathrm{x}=1}=\left[\frac{d}{d \eta}\left(\varphi^{\prime}(\eta)\right)\right] \varphi^{\prime}(\eta)=1=0
$$

Again

$$
\begin{gathered}
h^{\prime}(\mathrm{x})=\frac{d h}{d x}=\frac{\varphi^{\prime \prime \prime}}{\varphi^{\prime \prime}}=-\frac{\varphi^{\prime \prime}}{\varphi^{\prime \prime}}=-1 \\
\therefore h^{\prime}(0)=-1
\end{gathered}
$$

Substituting for $\mathrm{h}(\mathrm{x})=\varphi^{\prime \prime}$ and $\mathrm{x}=\varphi^{\prime}(\eta)$ then equation (12)and (13) takes the following form

$$
\begin{aligned}
& \mathrm{h} h^{\prime \prime}+\left(h^{\prime}\right)^{2}+h^{\prime}=0,0<\mathrm{x}<1 \\
& h^{\prime}(0)=-1 \text { and } \mathrm{h}(1)=0
\end{aligned}
$$

Equation (16) is a second order singular nonlinear boundary value problem. For $\alpha=1$ and $\beta=0.5$, equation (8) with boundary conditions (9) may be written as

$$
\begin{aligned}
& h^{2} h^{\prime \prime}-\frac{1}{2}\left(1-x^{2}\right) h^{\prime}=0,0<\mathrm{x}<1 \\
& h^{\prime}(0)=-0.5 \text { and } h(1)=0
\end{aligned}
$$

By setting $\mathrm{h}=f^{\prime \prime}(\eta)$ and $\mathrm{x}=f^{\prime}(\eta)$ and its positive solution has been studied by Shin [4]. The nonlinear differential equation (8) with boundary conditions (9) has been studied by many authors for different values of $\alpha$ and $\beta$ using different methods.

For $\alpha=0$ and $\beta=1$, equation (8) with boundary conditions (9) may be written as

$$
h^{2} h^{\prime \prime}+\left(h^{\prime}\right)^{2}-2 x=0,0<\mathrm{x}<1
$$




$$
h^{\prime}(0)=-0.86603 \text { and } \mathrm{h}(1)=0
$$

By letting $\mathrm{h}=f^{\prime \prime}(\eta)$ and $\mathrm{x}=f^{\prime}(\eta)$ and its positive solution has been studied by Molla[13].

The equation (16) is equivalent to the nonlinear differential equation (12) with boundary conditions (13). So positive solution of $(16)$ on $[0,1]$ is equivalent to the shear stress $f^{\prime \prime}(\eta)$ on $[0, \infty)$.

Let us define the following definition:

A function $\alpha_{1} \in C^{2}[0,1]$ is called a positive upper solution of (16), if $\alpha_{1}>0$ on $(0,1)$.

$$
\begin{gathered}
\alpha_{1} \alpha_{1}{ }^{\prime \prime}{ }_{+} \alpha_{1}{ }^{2}{ }^{2}+\alpha_{1}{ }^{\prime} \leq 0 \text { on }(0,1) . \\
\alpha_{1}^{\prime}\left((0) \leq-1 \text { and } \alpha_{1}(1) \geq 0\right.
\end{gathered}
$$

A function $\alpha_{2} \in C^{2}[0,1]$ is called a positive lower solution of (16), if $\alpha_{2}>0$ on $(0,1)$

$$
\begin{gathered}
\left.\alpha_{2} \alpha_{2}{ }^{\prime \prime}{ }_{+} \alpha_{2}\right)^{\prime}{ }^{2}+\alpha_{2}{ }^{\prime} \geq 0 \text { on }(0,1) \\
\alpha_{1}^{\prime}\left((0) \leq-1 \text { and } \alpha_{1}(1) \leq 0\right.
\end{gathered}
$$

Similar definitions hold for positive upper and lower solutions of a perturbation (19) which will be given in the following section.

A function $\mathrm{h} \in \mathrm{C}[0,1] \cap C^{2}[0,1)$ is called a positive solution of (16), if $h>0$ on $(0,1)$

$$
\begin{gathered}
h h^{\prime \prime}+\left(h^{\prime}\right)^{2}+h^{\prime}=0 \text { on }(0,1) \\
h^{\prime}(0)=-1 \text { and } h(1)=0
\end{gathered}
$$

\section{Establishing the Existence of Positive Solution}

Consider the non-linear boundary value problem

$$
\begin{aligned}
& h h^{\prime \prime}+\left(h^{\prime}\right)^{2}+h^{\prime}=0,0<\mathrm{x}<1 \\
& h^{\prime}(0)=-1 \text { and } h(1)=1 / p
\end{aligned}
$$

For each $\mathrm{p} \geq 1$, which may be viewed as a perturbation of (16).

We would like to prove, $h_{l p}(x)=0.01 x^{\alpha} \sqrt{1-x}+\frac{1}{p \alpha}$ is a positive lower solution of (19). For each $\mathrm{p} \geq 1$, where $\alpha=10^{k}$ and $\mathrm{k}$ is integer, finite but very large.

It is clear that $h_{l p}(x)>0$ on $(0,1), h_{l p}{ }^{\prime}(0)=0 \geq-1$, $h_{l p}(1)=\frac{1}{\alpha p}$ which can be written as $h_{l p}(1)=\frac{1}{\alpha p} \leq \frac{1}{p}$ and $\mathrm{g}(\mathrm{x})=h h^{\prime \prime}+\left(h^{\prime}\right)^{2}+h^{\prime}=h_{l p} h_{l p}{ }^{\prime \prime}+\left(h_{l p}{ }^{\prime}\right)^{2}+h_{l p}{ }^{\prime} \geq 0$, for $0<\mathrm{x}<1$ and $\mathrm{p} \geq 1$.

Thus $h_{l p}$ is a positive lower solution of (19). Accordingly $h_{l}=0.01 x^{\alpha} \sqrt{1-x}$ is a positive lower solution of (16). Again we have to prove, $h_{u p}(x)=2 \sqrt{1-x}+\frac{1}{p}$ is a positive upper solution of (19), for each $\mathrm{p} \geq 1$.

It is clear that $h_{u p}(x)>0$ on $(0,1), h_{u p}{ }^{\prime}(0)=-1$, which can be written as $h_{u p}{ }^{\prime}(0)=-1 \leq-1$ and $h_{u p}(1)=\frac{1}{p} \geq$

$$
\begin{aligned}
& \frac{1}{p} \cdot h_{l p} h_{l p}{ }^{\prime \prime}+\left(h_{l p}{ }^{\prime}\right)^{2}+h_{l p}{ }^{\prime}=-\left\{2 \sqrt{1-x}+\frac{1}{p}\right\}\{0.5(1- \\
& \left.x)^{\frac{3}{2}}\right\}+\frac{1}{1-x}-\frac{1}{\sqrt{1-x}} \leq 0
\end{aligned}
$$$$
\text { For, } 0<\mathrm{x}<1 \text { and } \mathrm{p} \geq 1
$$

Thus $h_{u p}$ is a positive upper solution of (19). Therefore, $h_{u}=2 \sqrt{1-x}$ is a positive upper solution of (16).

From Schauder's Fixed Point Theorem we know that, for any $\mathrm{p} \geq 1$, there exists a positive solution $h_{p} \in C^{2}[0,1]$ of the problem (19) such that $h_{l p} \leq h_{p} \leq h_{u p}$ on $0 \leq x \leq 1$.

Also again we have to prove $h_{p}=(1-x)+\frac{1}{p}$ is a positive solution of (19) for each $\mathrm{p} \geq 1$.

It is clear that $h_{p}(x)>0$ on $(0,1), h_{p}{ }^{\prime}(0)=-1, h_{p}(1)=$ $\frac{1}{p}$ and $h h^{\prime \prime}+\left(h^{\prime}\right)^{2}+h^{\prime}=h_{p} h_{p}{ }^{\prime \prime}+\left(h_{p}{ }^{\prime}\right)^{2}+h_{p}{ }^{\prime}=0$, for $0<x<1$ and $\mathrm{p} \geq 1$.

Thus $h_{p}$ is a positive solution of (19). Consequently $h=1-x$ is a positive solution of (16).

For any $\mathrm{p} \geq 1$, there exists a positive solution $h \in C^{2}[0,1]$ of the problem (16) such that $h_{l} \leq h \leq h_{u}$ on $0 \leq x \leq 1$, where $h_{l}, h$ and $h_{u}$ are as given above.

\section{Figures and Tables}

Now from the above discussion we have to make the tables and figures for different value of tangential velocity. With the help of figures and tables we discussed the shear stress for Homman and Convergent flow arising in the boundary layer theory.

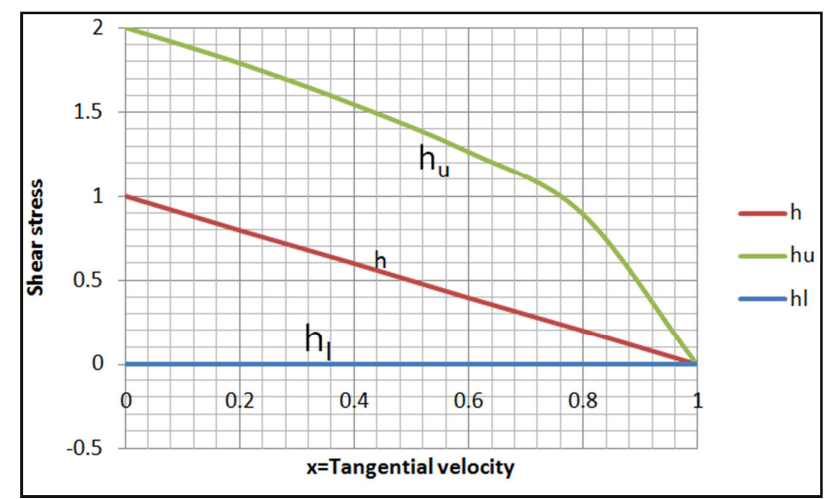

Figure 1. Different solutions such as $h, h_{l}$ and $h_{u}$. All are taken for even decimal number of tangential velocity.

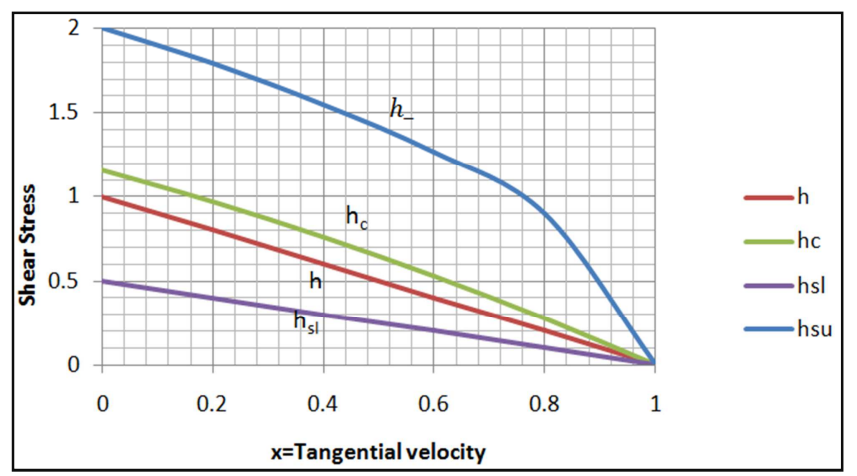

Figure 2. Different solutions such as $h, h_{c}, h_{s l}$ and $h_{s u}$. All are taken for even decimal number of tangential velocity. 
Table 1. Numerical values of different positive, positive lower and positive upper solutions with even decimal number.

\begin{tabular}{|c|c|c|c|c|c|}
\hline $\mathbf{x}$ & $h=1-x$ & $h_{c}=\sqrt{\frac{4-6 x+2 x^{3}}{3}}$ & $\mathbf{h}_{\mathrm{sl}}=(1-\mathrm{x}) / 2$ & $h_{1}=0.01 x^{\alpha} \sqrt{1-x}$ For $k=1$ & $h_{\mathrm{su}}=\mathbf{h}_{\mathrm{u}}=2 \sqrt{ }(1-x)$ \\
\hline 0 & 1 & 1.1547 & 0.05 & 0 & 2.000 \\
\hline 0.2 & 0.8 & 0.9688 & 0.04 & $9.1589 \mathrm{E}-10$ & 1.789 \\
\hline 0.4 & 0.6 & 0.7589 & 0.03 & $8.1222 \mathrm{E}-10$ & 1.549 \\
\hline 0.6 & 0.4 & 0.5266 & 0.02 & $3.8242 \mathrm{E}-05$ & 1.265 \\
\hline 0.8 & 0.2 & 0.2733 & 0.01 & 4.8019E-04 & 0.894 \\
\hline 1.0 & 0 & 0 & 0 & 0 & 0 \\
\hline
\end{tabular}

Table 2. Numerical values of different positive, positive lower and positive upper solutions with odd decimal number.

\begin{tabular}{llllll}
\hline $\mathbf{x}$ & $\mathbf{h}=\mathbf{1 - \mathbf { x }}$ & $\mathbf{h}_{\mathbf{c}}=\sqrt{\frac{4-\mathbf{6 x + 2 x ^ { 3 }}}{\mathbf{3}}}$ & $\mathbf{h}_{\mathbf{s l}}=(\mathbf{1 - x}) \mathbf{2}$ & $\mathbf{h}_{\mathbf{l}}=\mathbf{0 . 0 1} \boldsymbol{x}^{\alpha} \sqrt{\mathbf{1 - x}} \mathbf{F o r ~} \mathbf{k}=\mathbf{1}$ & $\mathbf{h}_{\mathrm{su}}=\mathbf{h}_{\mathrm{u}}=\mathbf{2} \sqrt{(\mathbf{1}-\boldsymbol{x})}$ \\
\hline 0 & 1 & 1.154701 & 0.5 & 0 & 2 \\
0.1 & 0.9 & 1.064894 & 0.45 & $9.49 \mathrm{E}-13$ & 1.897367 \\
0.3 & 0.7 & 0.866795 & 0.35 & $4.94 \mathrm{E}-08$ & 1.67332 \\
0.5 & 0.5 & 0.645497 & 0.25 & $6.91 \mathrm{E}-06$ & 1.414214 \\
0.7 & 0.3 & 0.402492 & 0.15 & 0.000155 & 1.095445 \\
0.9 & 0.1 & 0.139044 & 0.05 & 0.001103 & 0.632456 \\
1.0 & 0 & 0 & 0 & 0 & 0 \\
\hline
\end{tabular}

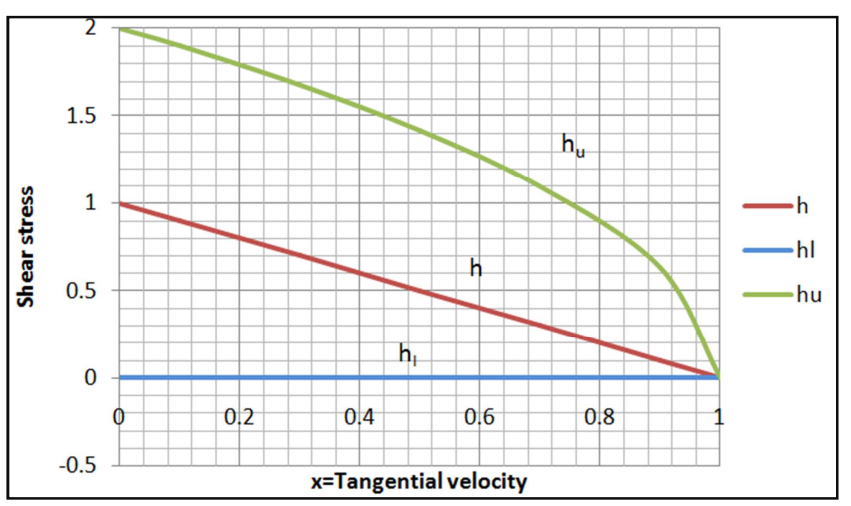

Figure 3. Different solutions such as $h, h_{l}$ and $h_{l}$. All are taken for odd decimal number of tangential velocity.

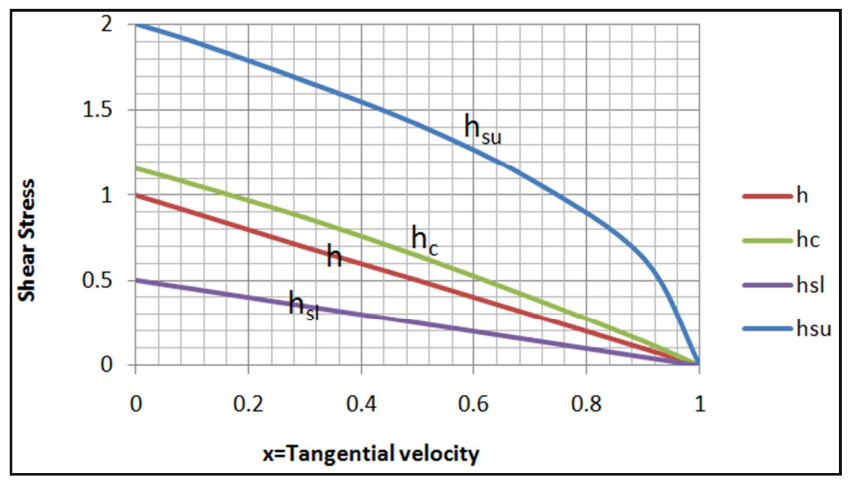

Figure 4. Different solutions such as $h, h_{c}, h_{s l}$ and $h_{\text {su. }}$. All are taken for odd decimal number of tangential velocity.

Table 3. Numerical values of different positive, positive lower and positive upper solutions with even and odd decimal number.

\begin{tabular}{llllll}
\hline $\mathbf{X}$ & $\mathbf{h}=\mathbf{1 - \mathbf { x }}$ & $\mathbf{h}_{\mathbf{c}}=\sqrt{\frac{4-\mathbf{6} \boldsymbol{x + 2 \boldsymbol { x } ^ { 3 }}}{\mathbf{3}}}$ & $\mathbf{h}_{\mathbf{s l}}=(\mathbf{1}-\mathbf{x}) / \mathbf{2}$ & $\mathbf{h}_{\mathbf{l}}=\mathbf{0 . 0 1} \boldsymbol{x}^{\alpha} \sqrt{\mathbf{1}-\boldsymbol{x}} \mathbf{F o r ~ k = 1}$ & $\mathbf{h}_{\mathrm{su}}=\mathbf{h}_{\mathrm{u}}=\sqrt{(\mathbf{1}-\boldsymbol{x})}$ \\
\hline 0 & 1 & 1.154701 & 0.5 & 0 & 2 \\
0.1 & 0.9 & 1.064894 & 0.45 & $9.49 \mathrm{E}-13$ & 1.897367 \\
0.2 & 0.8 & 0.968848 & 0.4 & $9.16 \mathrm{E}-10$ & 1.788854 \\
0.3 & 0.7 & 0.866795 & 0.35 & $4.94 \mathrm{E}-08$ & 1.67332 \\
0.4 & 0.6 & 0.758947 & 0.3 & $8.12 \mathrm{E}-07$ & 1.549193 \\
0.5 & 0.5 & 0.645497 & 0.25 & $6.91 \mathrm{E}-06$ & 1.414214 \\
0.6 & 0.4 & 0.526624 & 0.2 & $3.82 \mathrm{E}-05$ & 1.264911 \\
0.7 & 0.3 & 0.402492 & 0.15 & 0.000155 & 1.095445 \\
0.8 & 0.2 & 0.273252 & 0.1 & 0.00048 & 0.894427 \\
0.9 & 0.1 & 0.139044 & 0.05 & 0.001103 & 0.632456 \\
1.0 & 0 & 0 & 0 & 0 & 0 \\
\hline
\end{tabular}




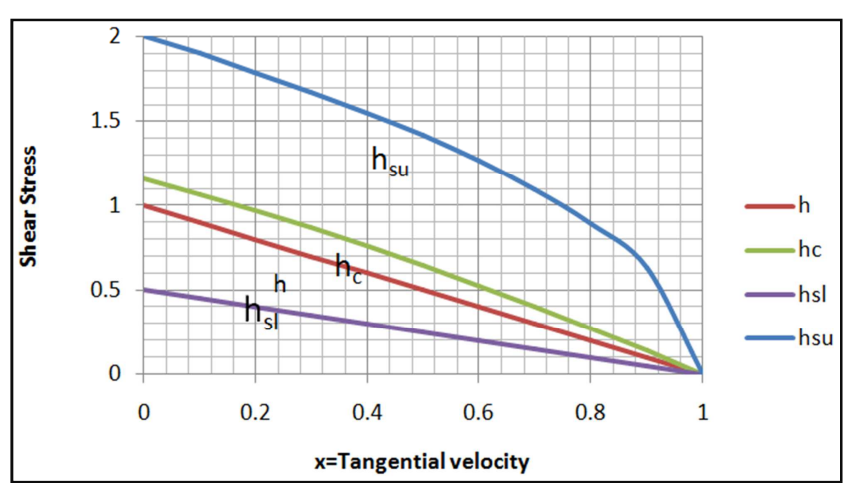

Figure 5. Different solutions such as $h, h_{c}, h_{s l}$ and $h_{s u}$. All are taken for both even and odd decimal number of tangential velocity.

\section{Results and Discussions}

The positive solution $h_{s}$ of (17) lies between the positive lower and upper solutions $\mathrm{h}_{\mathrm{sl}}=0.5(1-\mathrm{x})$ and $\mathrm{h}_{\mathrm{su}}=2 \sqrt{ }(1-x)$ respectively obtained by $\operatorname{Shin}[4]$. The positive solution $h_{c}$ $=\sqrt{\left(\frac{4-6 x+2 x 3}{3}\right)}$ of (18) lies between the positive lower and upper solutions $\quad \mathrm{h}_{\mathrm{lp}}=\quad \sqrt{ }(1.3334-2.0000705 x+$ $\left.0.0000035 x^{2}+0.666667 x^{3}\right)$ and $\mathrm{h}_{\text {up }}=3 \log (2-x)+3$ respectively obtained by Molla[13]. The positive solution of (16) is $\mathrm{h}=1-\mathrm{x}$ obtained by Molla [15]. For even decimal number of tangential velocity we get the Table 1 and both fig. 1 and fig. 2 which was done by Molla[15]. From the Table 2 and fig. 3 we see that the positive solution $\mathrm{h}$ of (12) lies between $h_{l}$ and $h_{u}$ for odd order decimal number of tangential velocity. Shin[4] shown that there will be exist a positive solution of (17) lies on between $\mathrm{h}_{\mathrm{sl}}$ and $\mathrm{h}_{\mathrm{su}}$. Also from the fig. 4 it is observed that the positive solution $h$ of (12) is less than or equal to the positive solution $h_{c}$ of (18) and the positive solution $h_{s}$ of (17) less than or equal to the positive solutions $h$ and $h_{c}$ of (12) and (18) respectively for odd decimal number of tangential velocity. From Table 2 it is clear that the shear stress for the strong suction is decreasing uniformly with increasing tangential velocity. On the other hand the shear stress for the convergent flow is decreasing not uniformly with increasing tangential velocity. From Table 3 and fig. 5 for both even and odd decimal number of tangential velocity, we see that the shear stress for the strong suction is decreasing uniformly with increasing tangential velocity. Also we get the shear stress for the convergent flow is decreasing not uniformly with increasing tangential velocity. For odd and both even and odd decimal number of the tangential velocity we get the result in fig. 3, fig. 4 , fig. 5 which is same as the result by Molla[15] in fig. 1, fig. 2 .

\section{Conclusion}

We have tried to discuss the shear stress between Homann and convergent flow for odd order decimal number and both even and odd order decimal number of the tangential velocity. By this study we have discussed positive solution, Homann flow, convergent flow, shear stress, tangential velocity etc. Also we have compared the shear stress of the strong suction with the shear stress of the Homann and Convergent flow. From the above figure we see that the obtained result is same as the previous result, i. e. It's a comparison between present and previous result.

\section{Acknowledgement}

Authors are grateful to the Department of Applied Mathematics, University of Rajshahi, Bangladesh for giving all facilities and support to carry out this work.

\section{References}

[1] Schmidt K., 1970. A nonlinear boundary value problem, J. Differential Equations 7, 527-537.

[2] Soewono E., K. Vajravelu and R. N. Mohapatra, 1991. Existence and nonuniqueness of solutions of a singular nonlinear boundary-layer problem, J. Math. Anal. Appl. 159, 251-270.

[3] Vajravelu K., E. Soewono and R. N. Mohapatra, 1991. On solutions of some singular, non-linear differential equations arising in boundary layer theory, J. Math. Anal. Appl. 155, 499-512.

[4] Shin J. Y., 1997. A Singular nonlinear differential equation arising in the Homann flow, J. Math. Anal. Appl. 212, 443-451.

[5] Schlichting H., and K. Gersten, 1999. Boundary Layer Theory, Springer, 113, 114.

[6] Shanti Swarup, 2000. Fluids Dynamics, Krishna Prakashan Media(P) Ltd. Merut, 622.632.633.

[7] Molla M. R. and S. Banu, 2003. Some singular nonlinear BVPS arising in the boundary layer flow. Ganit: Journal of Bangladesh Mathematical Soc., 23, 91-103.

[8] Molla M. R., 2005. Existence and Uniqueness of positive solution of the suction of the fluid from the boundary layer, J. Math. and Math. Sci, JU, Savar, Bangladesh, 20, 31-40.

[9] Molla M. R. and S. Banu, 2006. Existence and uniqueness of positive solution of a singular nonlinear BVP. Journal of Science, University of Dhaka, 54 (2), 191-195.

[10] Molla M. R., 2008. A singular non-linear BVP arising in the boundary layer flow along a flat plate, Ganit: J. Bangladesh Math. Soc. 28, 59-67.

[11] Molla M. R., M. K. Jaman and M. Hasan, 2011. Comparison of positive solutions for two boundary value problems arising in the boundary layer flow. Journal of Science, University of Dhaka, 59 (2), 167-172.

[12] Molla M. R., 2012. A singular non-linear boundary value problem arising in a convergent channel, Bangladesh Journal of Physics, 12, 15-26.

[13] Molla M. R. and M. Begum, 2012. Existence and Uniqueness of positive solution of the injection of the fluid into the boundary layer. Jahangirnagar J. of Math. and Math. Sciences, 27, 103-114. 
[14] Molla M. R., 2013. An analytic treatment of the Falkner-Skan boundary layer equation. Journal of Science, University of Dhaka, 61(1), 139-144.
[15] Molla M. R., 2014. Comparison of Shear Stresses of Different Fluids Flows Arising in the Boundary Layer Theory. Dhaka Univ. J. Sci. 62(2): 115-118. 